\title{
Clinical ethnography in severe mental illness: a clinical method to tackle social determinants and structural racism in personalised care
}

\author{
Kamaldeep Bhui, Simon Dein and Catherine Pope
}

Ethnic inequalities in the experiences and outcomes of severe mental illness are well established. These include a higher incidence of severe mental illnesses (psychoses), adverse pathways into and through care, including crisis care, police and criminal justice systems involvement, and care under the powers of the Mental Health Act. The situation persists despite awareness and is driven by a mixture of the social determinants of poor health, societal disadvantage and structural racism, as well as conflictual interactions with care systems, which themselves are configured in ways that sustain or deepen these inequalities. Although training and education are often proposed, this is not shown to have sustained effects. Clinical processes (interviewing/assessment/formulation/intervention) need to address systemic influences and improve the cultural precision with which care is delivered, organised and commissioned. We discuss clinical ethnography and present evidence of its value in addressing systemic as well as individual care needs for diverse communities.

\section{Keywords}

Ethnicity; severe mental illness; racism; ethnography; inequalities.

\section{Copyright and usage}

(C) The Author(s), 2021. Published by Cambridge University Press on behalf of the Royal College of Psychiatrists. This is an Open Access article, distributed under the terms of the creative Commons Attribution-NonCommercial licence (http://creative commons.org/licenses/by-nc/4.0/), which permits non-commercial re-use, distribution, and reproduction in any medium, provided the original work is properly cited. The written permission of Cambridge University Press must be obtained for commercial re-use.
Kamaldeep Bhui is Professor of Psychiatry at the University of Oxford. Simon Dein is Honorary Professor of Anthropology and Psychiatry at the Centre for Psychiatry, Barts \& the London School of Medicine and Dentistry, Queen Mary University of London, and a consultant psychiatrist, and former general practitioner. Catherine Pope is Professor of Medical Sociology at Nuffield Department of Primary Care Health Sciences at the University of Oxford and an expert in qualitative research methods. She is a senior research fellow at Green Templeton College and a Fellow of the Academy of Social Sciences

\section{Ethnic inequalities of severe mental illness}

Ethnic inequalities in experiences and outcomes of severe mental illness have been demonstrated for several decades, including a higher incidence of psychosis-related service contacts (Black Caribbean, Black African, South Asian, mixed heritage and migrants) and poorer mental and physical health outcomes related to racism. ${ }^{1-3}$ Personality disorder diagnoses are less common in Black patients in routine clinical practice, but there is less ethnic difference if more reliable structured assessments are used. ${ }^{4}$ Black Caribbean, Black African, South Asian (Indian, Pakistani, Bangladeshi), migrants and minoritised/racialised groups in general experience adverse pathways to care, including more crisis care delivered in emergency departments, and the police and criminal justice system, more care under the powers of the Mental Health Act, less access to effective psychotherapies and more pharmacological interventions. ${ }^{5,6}$ These inequalities may be considered inevitable consequences of the communication challenges across linguistic and cultural barriers but can be better understood as expressions of structural or institutional racism.

Racism is one of the many social determinants of poor health, but there is little action to surface direct and indirect consequences of racism, nor are there interventions to engage with patients' narratives of racism. Racism influences the lens through which people's abilities and hopes are seen and acted upon when minoritised groups engage with public services including housing, education, employment and welfare support, all of which may lead to more need for healthcare. If minoritised groups seek help for mental distress linked closely to social stressors (such as poor housing or poverty), their access to care may be hindered by the constraints those stressors place on their ability to seek help, engage with and use interventions. These social stressors are frequently set aside by practitioners and disconnected from healthcare and services in ways that prevent meaningful advocacy and social support during crises and periods of help-seeking.

If healthcare solutions proffered are themselves produced by practices that are blind to racism and also generate and perpetuate inequalities, patients may fear and avoid health services. Thus, clinical practice needs to incorporate assessment and intervention directed towards social determinants and structural racism. Expectations of care and health beliefs are one modifiable cause of conflict, relationship breakdowns and disengagement. ${ }^{7}$ Addressing health beliefs may avert frustration and conflict within therapeutic relationships ${ }^{8}$ and support treatment effectiveness. Some ethnically minoritised people consult traditional and religious healers who share spiritual models of mental distress and offer culturally congruent forms of help, but this process may delay access to formal health services. ${ }^{9}$ Furthermore, traditional and religious healers may help with depression and anxiety symptoms, but there is no evidence of benefits for people with bipolar disorder or psychosis. ${ }^{10}$

Up to $40 \%$ of people with severe mental illness, in need of ongoing care, disengage from community mental health services. Men, young people, some ethnic groups and people facing isolation, marginalisation and deprivation have higher rates of disengagement from mental health services. ${ }^{11,12}$ Those who disengage experience delays in receipt of effective care, and this may lead to no care, or more restrictive treatments if there is a subsequent crisis (including involuntary admissions) at a greater cost to the National Health Service (NHS). There are also additional risks of suicide and 
violence related to untreated mental illness, and attendant criminal justice system involvement. Dangerous stereotypes of Black patients may perpetuate coercive care and over-restrictive practices that are in themselves dangerous, extending concerns about the safety of services. ${ }^{13}$

How should practitioners and service providers, not to mention commissioners and policymakers respond? There are many public health, and early preventive actions that can be taken to protect people from adverse experiences and to challenge discrimination. Yet, clinicians encountering patients with complex problems lack meaningful and practical approaches that consider wider systemic harms, such as racism, and improve practice and outcomes. A systematic review of therapeutic communications in ethnically minoritised populations demonstrated the importance of ethnography, among the many approaches that seek to improve cultural competence, capability and the effectiveness of healthcare interventions. ${ }^{14}$

\section{Ethnography as a clinical and systemic intervention}

Ethnographic research methods are used in social science to gather knowledge that attends to narratives (or stories) and beliefs. In this paper we use the term clinical ethnography and highlight the use of ethnographic methods in clinical interviewing and care delivery

Dominicé Dao et $\mathrm{al}^{15}$ define clinical ethnography thus: '.. encourages clinicians to explore the patient's explanatory model of illness, recourse to traditional and alternative healing practices, healthcare expectations and social context, and to use this information to negotiate a mutually acceptable treatment plan'.

Calabrese's definition ${ }^{16}$ expands on this: ' $\ldots$ culturally- and clinically-informed self-reflective immersion in local worlds of suffering, healing, and wellbeing to produce data that is of clinical as well as anthropological value'.

Ethnography in research may use participant observations, informal conversations, semi-structured interviews and the collection of visual data such as photographs and recordings to reveal a person's narratives, perspectives, values and the way people organise their experiences to create meaning and coherence, taking account of family, and cultural and religious norms. Ethnographic methods have also been used to study clinical practice and health systems, notably in rapid ethnographies that seek to improve understanding of care needs, improve services, and inform policy and commissioning. ${ }^{17,18}$

Clinical ethnography harnesses the ethnographic practice of interviewing and uncovering subjective meaning and relocates this in clinical endeavours to enhance the clinician's understanding of the patient's world view, and their 'emic' or insider perspectives. This can include gathering knowledge about cultural heritage, health beliefs and biographies, to inform adaptation of care practices for immigrants, minoritised and indigenous people. The objective is to use this deeper understanding to improve the care offered, adapting it as needed, to the context of the patient.

The proposed method of clinical enquiry is empowering with beneficial outcomes in a diverse range of conditions and patient populations. ${ }^{14,19,20}$ The theory of change differs from behaviour change interventions that rely on cognitions, for example the health belief model, theories of reasoned action, planned behaviour and transtheoretical models by taking much greater account of the wider systemic social, economic and cultural constraints. ${ }^{21,22}$ In contrast, clinical ethnography considers systemic factors that influence onset and recovery from mental illness, given its roots in social anthropology and attention to kinship, economics and cultural knowledge, actions and behaviours. ${ }^{8,19}$

Clinical ethnography also encourages power sharing and gives prominence to the patient's voice in the consultation, engendering trust, motivating help-seeking, and improving the quality of care, engagement and outcomes for culturally diverse patients in mental health services. ${ }^{8,20,23-26}$ Non-randomised studies show the approach leads to better diagnosis and treatment, improved outcomes in psychosis, ${ }^{8,14}$ as well as uptake of, and better outcomes in psychotherapy for depression. ${ }^{25}$ In previous work, we successfully adapted a clinical ethnographic approach for use in NHS mental healthcare, making use of specialist cultural consultation experts working alongside care workers. ${ }^{19}$ This intervention involved assessing identity, reconciling the cultural health beliefs and expectations of the patient (using Barts Explanatory Model Interview), their social and cultural group, and including care workers' reflections on their own health beliefs. ${ }^{19}$ Referrals were from care professionals or patients themselves, and were driven by conflict or uncertainty about how to tailor care to take account of race, ethnicity and culture. Racism was a feature of patients' experiences of services, and why professionals sought guidance, fearing they were not skilled at dealing with such matters. The service gathered patient narratives, and took account of psychosocial and structural factors, including racism as a form of trauma, that affected mental state, help-seeking and fear of coercion. The service also equipped clinicians with the necessary vocabularies, insider knowledge and summaries of epidemiological and sociocultural research of relevance to specific patients or patient groups. This meant that the service was able to provide useful care recommendations, for people living with psychoses, mostly receiving care from assertive outreach teams or while admitted to hospital. The outcomes included fewer contacts with assertive outreach teams, and consequential savings at 3 -month follow-up, ${ }^{19}$ and more confidence in holding such conversations among the professionals. Despite the emerging evidence of value, no NHS commissioned services have adopted this specialist cultural consultation and ethnographic approach. The principles of cultural consultation are being re-discovered, recognised and applied, driven by the interests of specific practitioners rather than by a comprehensive drive to ensure quality standards are devised or met.

To summarise, clinical ethnography permits reflexivity in clinical encounters and opens up the possibility that the clinician's own values and constructions of race/ethnicity and culture can be considered alongside learning about the social and cultural context of distress, and its expression in a specific patient, with a specific ethnic/racial heritage and identity. The ethnographic interview facilitates the development of rapport and trust and seeks a collaborative process for gathering information, weighing it up and choosing therapeutic actions. In skilled hands, the approach should reduce the power differentials, correct misunderstandings and repair therapeutic ruptures within an ongoing formative process.

\section{Clinical Ethnography and COVID-19}

The coronavirus disease 2010 (COVID-19) pandemic has revealed and escalated health inequalities, especially for racialised and ethnically minoritised people. Yet, there have been few ethnographies of the challenges. Recent rapid ethnographies of COVID contexts in Macau and USA show how a narrow biomedical and quarantine-focused narrative can undermine broader health and wellbeing; and these studies reveal difficulties for patients and for clinicians in adapting to new routines and infection-prevention priorities. $^{27,28}$ Stark differences in the spread of COVID-19 and in health outcomes have exposed how racism engenders and reinforces these inequalities. As a consequence, there is a growing awareness that we must adapt health and social care to be, not only culturally competent, but also capable of tackling fundamental causes of inequalities, both the social determinants and racism. ${ }^{29}$ 
Understanding of the biology of the virus must be pursued alongside interrogation of the political and social contexts of the pandemic. ${ }^{30}$ History shows that exclusionary and xenophobic sentiments emerge at the time of pandemics, and these can be as damaging as the virus by exposing and deepening divisions and inequality. ${ }^{31}$ Clinical ethnography and broader social sciences approaches such as critical anthropology can challenge the narrow biomedical and structurally bereft lens through which we continue to make mistakes of the past at the levels of policy and practice. ${ }^{32}$

Kamaldeep Bhui (D), Department of Psychiatry and Nuffield Department of Primary Care Health Sciences, University of Oxford, UK; East London NHS Foundation Trust, UK; and World Psychiatric Association Collaborating Centre, UK; Simon Dein, Queen Mary University of London, UK; Catherine Pope, Nuffield Department of Primary Care Health Sciences, University of Oxford, UK

Correspondence: Kamaldeep Bhui. Email: kam.bhui@psych.ox.ac.uk

First received 20 Feb 2021, final revision 16 Mar 2021, accepted 17 Mar 202

\section{Author contributions}

K.B. conceived of the paper and wrote the first draft. S.D. and C.P. have reviewed the manuscript and made significant contributions to the revisions and in response to reviewers. All authors approve the final draft.

\section{Funding}

The content of this paper received no specific grant from any funding agency, commercial or not for profit. The work cited on cultural consultation and systematic reviews was funded by Tower Hamlets Clinical Commissioning Group and National Institute for Health Research Health Technology Assessment respectively, with K.B. as principal investigator.

\section{Declaration of interest}

K.B. is Editor-in-Chief of BJPSych and on the board of BJPsych Open but has not taken part in the decision-making process or reviews of this paper. The authors declare there are no other conflicts of interest.

\section{References}

1 Paradies Y, Ben J, Denson N, Elias A, Priest N, Pieterse A, et al. Racism as a determinant of health: a systematic review and meta-analysis. PLOS One 2015; 10: e0138511.

2 Priest $\mathrm{N}$, Paradies $\mathrm{Y}$, Trenerry B, Truong M, Karlsen S, Kelly Y. A systematic review of studies examining the relationship between reported racism and health and wellbeing for children and young people. Soc Sci Med 2013; 95: $115-27$.

3 Halvorsrud K, Nazroo J, Otis M, Brown Hajdukova E, Bhui K. Ethnic inequalities in the incidence of diagnosis of severe mental illness in England: a systematic review and new meta-analyses for non-affective and affective psychoses. Soc Psychiatry Psychiatr Epidemiol 2019; 54: 1311-23.

4 McGilloway A, Hall RE, Lee T, Bhui KS. A systematic review of personality disorder, race and ethnicity: prevalence, aetiology and treatment. BMC Psychiatry 2010; 10: 33.

5 Halvorsrud K, Nazroo J, Otis M, Brown Hajdukova E, Bhui K. Ethnic inequalities and pathways to care in psychosis in England: a systematic review and metaanalysis. BMC Med 2018; 16: 223.

6 Das-Munshi J, Bhugra D, Crawford MJ. Ethnic minority inequalities in access to treatments for schizophrenia and schizoaffective disorders: findings from a nationally representative cross-sectional study. BMC Med 2018; 16: 55.

7 Owiti JA, Palinski A, Ajaz A, Ascoli M, De Jongh B, Bhui KS. Explanations of illness experiences among community mental health patients: an argument for the use of an ethnographic interview method in routine clinical care. Int Rev Psychiatry 2015; 27: 23-38

8 Kirmayer LJ, Groleau D, Guzder J,Blake C, Jarvis E. Cultural consultation: a model of mental health service for multicultural societies. Can J Psychiatry 2003; 48: 145-53.

9 Dein S. Religious healing and mental health. Ment Health Relig Cult 2020; 23: 657-65.
10 van der Watt ASJ, van de Water T, Nortje G, Oladeji BD, Seedat S, Gureje O, et al. The perceived effectiveness of traditional and faith healing in the treatment of mental illness: a systematic review of qualitative studies. Soc Psychiatry Psychiatr Epidemiol 2018; 53: 555-66.

11 O'Brien A, Fahmy R, Singh SP. Disengagement from mental health services. A literature review. Soc Psychiatry Psychiatr Epidemiol 2009; 44: 558-68.

12 Oflaz S, Guveli H, Kalelioglu T, Akyazı S, Yıldızhan E, Kılıc KC, et al. Illness perception of dropout patients followed up at bipolar outpatient clinic, Turkey. Asian J Psychiatr 2015; 15: 5.

13 Lipsedge M. Dangerous stereotypes. J Forens Psychiatry 1994; 5: 14-9.

14 Bhui KS, Aslam RW, Palinski A, McCabe R, Johnson MR, Weich S, et al. Interventions to improve therapeutic communications between Black and minority ethnic patients and professionals in psychiatric services: systematic review. Br J Psychiatry 2015; 207: 95-103.

15 Dominicé Dao $M$, Inglin S, Vilpert S, Hudelson P. The relevance of clinical ethnography: reflections on 10 years of a cultural consultation service. BMC Health Serv Res 2018; 18: 19.

16 Calabrese JD. A Different Medicine: Postcolonial Healing in the Native American Church. Oxford University Press, 2013.

17 Vindrola-Padros C, Vindrola-Padros B. Quick and dirty? A systematic review of the use of rapid ethnographies in healthcare organisation and delivery. $B M J$ Qual Saf 2018; 27: 321.

18 Palinkas LA, Zatzick D. Rapid assessment procedure informed clinical ethnography (RAPICE) in pragmatic clinical trials of mental health services implementation: methods and applied case study. Adm Policy Ment Health Ment Health Serv Res 2019; 46: 255-70.

19 Bhui KS, Owiti JA, Palinski A, Ascoli M, De Jongh B, Archer J, et al. A cultural consultation service in East London: experiences and outcomes from implementation of an innovative service. Int Rev Psychiatry 2015; 27: 11-22.

20 Aggarwal NK, Desilva R, Nicasio AV, Boiler M, Lewis-Fernández R. Does the Cultural Formulation Interview for the fifth revision of the diagnostic and statistical manual of mental disorders (DSM-5) affect medical communication? A qualitative exploratory study from the New York site. Ethn Health 2015; 20 : $1-28$.

21 Davis R, Campbell R, Hildon Z, Hobbs L, Michie S. Theories of behaviour and behaviour change across the social and behavioural sciences: a scoping review. Health Psychol Rev 2015; 9: 323-44.

22 Krumeich A, Weijts W, Reddy P, Meijer-Weitz A. The benefits of anthropological approaches for health promotion research and practice. Health Educ Res 2001; 16: 121-30.

23 Rudell K, Bhui K, Priebe S. Concept, development and application of a new mixed method assessment of cultural variations in illness perceptions: Barts Explanatory Model Inventory. J Health Psychol 2009; 14: 336-47.

24 Owiti JA, Ajaz A, Ascoli M, de Jongh B, Palinski A, Bhui KS Cultural consultation as a model for training multidisciplinary mental healthcare professionals in cultural competence skills: preliminary results. J Psychiatr Ment Health Nurs 2014; 21: 814-26.

25 Grote NK, Swartz HA, Geibel SL, Zuckoff A, Houck PR, Frank E. A randomized controlled trial of culturally relevant, brief interpersonal psychotherapy for perinatal depression. Psychiatr Serv 2009; 60: 313-21.

26 Grote N, Zuckoff A, Hea S. Engaging women who are depressed and economically disadvantaged in mental health treatment. Social Work 2007; 52: 295308

27 Zuev D, Hannam K. Anxious immobilities: an ethnography of coping with contagion (Covid-19) in Macau. Mobilities 2021; 16: 35-50.

28 Palinkas LA, Whiteside L, Nehra D, Engstrom A, Taylor M, Moloney K, et al. Rapid ethnographic assessment of the COVID-19 pandemic April 2020 'surge' and its impact on service delivery in an acute care medical emergency department and trauma center. BMJ Open 2020; 10: e041772.

29 Hansen $\mathrm{H}$, Braslow J, Rohrbaugh RM. From cultural to structural competencytraining psychiatry residents to act on social determinants of health and institutional racism. JAMA Psychiatry 2018; 75: 117-8.

30 Hardy L. Connection, contagion, and COVID-19. Med Anthropol 2020; 39: 655-9.

31 Onoma AK. Epidemics, xenophobia and narratives of propitiousness. Med Anthropol 2020; 39: 382-97.

32 Panter-Brick C. Health, risk, and resilience: interdisciplinary concepts and applications. Annu Rev Anthropol 2014; 43: 431-8.

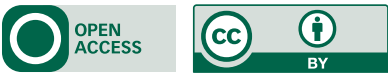

